\title{
Verbal instructional sets to normalise the temporal and spatial gait variables in Parkinson's disease
}

\author{
Andrea L Behrman, Philip Teitelbaum, James H Cauraugh
}

\begin{abstract}
Gait in Parkinson's disease is characterised by slowed velocity; shuffling, small steps; and absent arm swing. Drug therapy intervention is beneficial in improving mobility, though with prolonged use its effects may diminish. The purpose of this study was to examine whether Parkinsonian patients could improve their gait patterns in response to five instructional sets: natural walking; walking while deliberately swinging the arms; walking with large steps; fast walking; and walking while counting aloud. Eight subjects with idiopathic Parkinson's disease and eight age matched control subjects were tested using motion analysis. The findings indicated that parkinsonian patients followed the instructions which immediately altered a series of single walking variables. Simultaneously, automatically activated changes occurred in other gait variables producing more normal gait. The instructional set is a strategy which can aid normalisation of Parkinsonian gait although its benefits may depend on the stage of disease progression and the degree of attention to the instructions. (F Neurol Neurosurg Psychiatry 1998;65:580-582)
\end{abstract}

Keywords: Parkinson's disease; gait; instructional set; ambulation; hypokinesia

Psychology, PO Box

112250

P Teitelbaum

Department of Exercise and Sport

Sciences, Motor

Behavior Laboratory, PO Box 118206,

University of Florida,

Gainesville, Florida

32610, USA

J Cauraugh

Correspondence to: Dr Andrea L Behrman, Department of Physical Therapy, Motor Behavior Laboratory, PO Box 100154, University of Florida,

Gainesville, FL 32610, USA.

Received 1 July 1997 and in final revised form

9 March 1998

Accepted 16 March 1998

Patients with Parkinson's disease typically walk slowly, with short, shuffling steps, stooped posture, and little or no arm swing. ${ }^{12}$ Drug therapy is usually beneficial in improving walking function in such patients, although such benefits often decrease as the disease progresses. Researchers have reported temporary improvements in walking in patients with Parkinson's disease when they use visual targets, such as markers on the floor, with and without attentional strategies. ${ }^{3-5}$ Preliminary findings and anecdotal reports indicate that patients with Parkinson's disease can temporarily alter their walking pattern when attending to specific verbal instructions. ${ }^{126}$ The purpose of this study was to determine if persons with Parkinson's disease could improve their walking pattern by following specific instructions to alter a series of gait variables (for example, arm swing excursion, stride length). A further aim was to study concomitant changes in other gait variables as a basis for gait rehabilitation. We hypothesised that verbal instructions to increase the intensity of one variable of walking may result in the simultaneous augmentation of other biomechanically and neurologically linked gait variables. ${ }^{78}$

\section{Method}

SUBJECTS

Eight community dwelling elderly patients with idiopathic Parkinson's disease (mean (SD) age 72.9 (4.7) years and mean (SD) disease duration 11.6 (6.4) years) and eight age and sex matched adults without Parkinson's disease (mean (SD) age 72.7 (4.5) years), participated in this study. Each group consisted of six men and two women. Participants had no history of other neurological, mental, cardiac, musculoskeletal, or visual disorders which affected walking. Mental status screening with the mini mental state examination gave mean scores of 28.5 (SD 5.3) for the patients with Parkinson's disease and 29.2 (SD 1.1) for the control subjects. ${ }^{9}$ According to the Hoehn and Yahr scale, the disability level of two patients with Parkinson's disease was stage II, whereas four patients were at stage III, and two at stage IV. ${ }^{10}$ All participants with Parkinson's disease were on medication (sinemet) and were tested during self reported "on" periods within 1 to 2 hours after its administration.
APPARATUS

Participants walked across a $7.5 \mathrm{~m}$ indoor level path and were videotaped with a Panasonic AG camera at 1/1000 shutter speed. Standardised guidelines for two dimensional videotaping were followed to minimise camera misalignment and parallax. ${ }^{11}$ The camera view was perpendicular to the participant's left side and plane of motion. The camera distance from the walkway and the focal length were set to maximally fill the complete field of view with the subject. The focal length was adjusted to view a minimum of two complete gait cycles. The Peak Video Illustrator motion analysis system (Englewood, Colorado) was used to analyse spatial and temporal variables of gait by identifying the make and break point of foot contact and the simultaneous shoulder and elbow joint 
Mean (SD) of six gait variables for patients with Parkinson's disease (PD) and for controls for each instructional set

\begin{tabular}{llll}
\hline & & $P D$ & Controls \\
\hline Left step length $(\mathrm{m})$ & Natural walk & $0.85(0.27)$ & $1.19(0.19)$ \\
& Swing arms & $0.99(0.31)$ & $1.41(0.24)$ \\
& Count aloud & $0.98(0.32)$ & $1.31(0.20)$ \\
& Large steps & $1.26(0.20)$ & $1.56(0.15)$ \\
& Walk fast & $1.08(0.41)$ & $1.52(0.21)$ \\
Right step length (m) & Natural walk & $0.83(0.27)$ & $1.17(0.19)$ \\
& Swing arms & $0.98(0.29)$ & $1.37(0.23)$ \\
& Count aloud & $0.95(0.30)$ & $1.31(0.20)$ \\
& Large steps & $1.33(0.30)$ & $1.62(0.21)$ \\
Velocity (m/s) & Walk fast & $1.06(0.39)$ & $1.51(0.20)$ \\
& Natural walk & $0.77(0.23)$ & $1.02(0.23)$ \\
& Swing arms & $0.93(0.30)$ & $1.22(0.22)$ \\
& Count aloud & $0.88(0.31)$ & $1.18(0.29)$ \\
Large steps & $1.16(0.36)$ & $1.35(0.26)$ \\
& Walk fast & $1.27(0.38)$ & $1.70(0.37)$ \\
& Natural walk & $28(24)$ & $24(11)$ \\
& Swing arms & $61(38)$ & $52(21)$ \\
& Count aloud & $41(27)$ & $27(13)$ \\
& Large steps & $54(47)$ & $36(24)$ \\
& Walk fast & $36(31)$ & $43(18)$ \\
& Natural walk & $3(2)$ & $7(6)$ \\
& Swing arms & $8(6)$ & $21(9)$ \\
& Count aloud & $7(3)$ & $27(27)$ \\
& Large steps & $10(6)$ & $14(9)$ \\
& Walk fast & $16(8)$ & $27(10)$ \\
& Natural walk & $117(29)$ & $103(11)$ \\
& Swing arms & $112(12)$ & $103(9)$ \\
& Count aloud & $108(16)$ & $109(14)$ \\
& Large steps & $94(13)$ & $99(11)$ \\
& Walk fast & $153(46)$ & $133(18)$ \\
\hline & & &
\end{tabular}

positions. ${ }^{12-14}$ Data were collected from the middle $4 \mathrm{~m}$ of the walkway. ${ }^{3}$

\section{PROCEDURE}

Participants reviewed and signed an informed consent, as approved by the University of Florida Health Center Institutional Review Board. They were interviewed for medical history and tested for mental status and disability rating. Participants wore shorts, walked barefoot, and were videotaped using specified instructional sets. Single trials of natural walking were interspersed between four randomised, verbally instructed conditions: (1) walking while deliberately swinging the arms, (2) walking while counting aloud, (3) walking with large steps, or (4) fast walking.

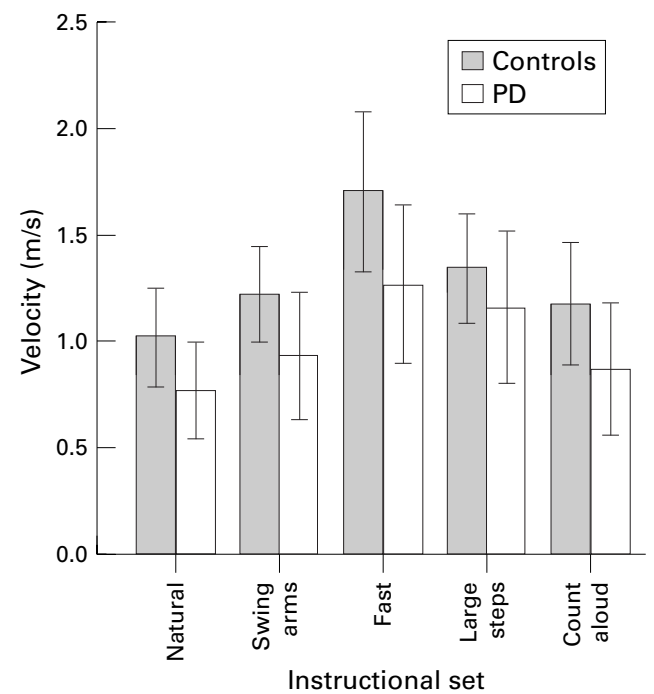

Mean (SD) gait velocities for control and Parkinson's disease groups for each instructional set: natural walk, walk with deliberate arm swing, walk fast, walk with large steps, and walk while counting aloud.
The videotapes were analysed to identify the values for five dependent variables: (1) right and left step length (m); (b) cadence (steps/ min), (c) left shoulder excursion; (d) elbow joint motion from toe off to heel strike of the right lower extremity; and (e) ambulation velocity $(\mathrm{m} / \mathrm{s})$.

DATA ANALYSIS

Separate analyses, $2 \times 5$ analyses of variance (ANOVAs) (group $\times$ instructional set) with repeated measures on the second factor, were performed for each gait variable. All analyses were performed with an alpha level set at 0.05 .

\section{Results}

Descriptive data on gait variables for each instructional set by groups are presented in table 1. The significant findings for the variables included a group effect for left step length $(F(1,14)=11.15)$, right step length $(F(1,14)=9.34)$, ambulation velocity $(F(1$, $14)=4.88)$, and elbow excursion $) F(1$, $14)=9.79)$. Compared with the control group, the left and right step lengths were $26 \%$ shorter, gait velocity $23 \%$ slower, and elbow excursion 53\% less for the Parkinson's disease group. Secondly, a significant effect was found for instructional set for right step length $(F(4$, $56)=32.95)$; left step length $(F(4,56)=14.10)$; ambulation velocity $(F(4,56)=35.5)$; cadence $(F(4,56)=12.96)$; shoulder excursion $(F(4$, $56)=8.49)$; and elbow excursion, $(F(4,56)=$ 6.36). There was no significant group $\times$ instructional set interaction for any of the gait variables.

In response to the instructional set, both groups were able to voluntarily alter a gait variable-for example, arm swing amplitudeand showed a significant change in that variable. In addition to the participants' response to single instructional sets, concomitant changes occurred in other gait variables. For instance, when instructed to walk while deliberately swinging the arms, both shoulder and elbow excursions increased $(118 \%$ and $197 \%$ respectively), walking velocity increased by $20 \%$, and step length increased by $18 \%$. When instructed to walk with large steps, the right and left step length increased by $47 \%$ and $38 \%$. Velocity concomitantly increased by $40 \%$ and arm swing excursion increased by $73 \%$, whereas cadence decreased by $14 \%$. When asked to walk fast, step lengths increased by $28 \%$, arm swing excursion (shoulder by $52 \%$ and elbow by $335 \%$ ) and cadence increased by $30 \%$. When asked to walk while counting aloud, only velocity increased significantly by $14 \%$. Lastly, when asked to walk fast, walking velocity increased in both groups by $65 \%$.

The pattern of change in mean gait velocity for the five instructional sets was similar for the two groups (figure). However, in response to the instructions for fast walking and large steps, both groups demonstrated significant increases in gait velocity from their natural walking velocity. Compared with their natural speed, the Parkinson's disease group increased their velocity $65 \%$ during fast walking and $50 \%$ when walking with large steps. The walking 
velocities of the Parkinson's disease group were comparable or surpassed the natural walking velocity of the control group $(1.02 \mathrm{~m} / \mathrm{s})$ for the three instructional sets: (1) while deliberately swinging the arms $(0.93 \mathrm{~m} / \mathrm{s})$, (2) walking with large steps $(1.16 \mathrm{~m} / \mathrm{s})$, and (3) fast walking $(1.27 \mathrm{~m} / \mathrm{s}$, figure).

\section{Discussion}

This study showed that the walking patterns of patients with Parkinson's disease improved in response to specific instructions to change a series of single gait variables. Patients with Parkinson's disease can intentionally walk with larger steps, faster, and with increased arm swing amplitude. Furthermore, by increasing the intensity of one gait variable-for example, arm swing amplitude-other gait variables improved. These findings indicate that cognitive strategies have the potential to improve the overall walking patterns of patients with Parkinson's disease.

This confirms previous findings that patients with Parkinson's disease respond to verbal instructions to alter their walking pattern. ${ }^{12}$ For example, Morris et al reported that patients with Parkinson's disease who focus deliberately on walking with normal stride length improve their walking patterns. ${ }^{5}$ They suggested that strategies employing instructional sets and deliberate attention to specific elements of "normal" walking may bypass basal ganglia circuitry and activate the frontal and prefrontal areas of the brain to prepare the motor cortex for locomotion. ${ }^{15}$ The use of this cognitive strategy may therefore provide an internal stimulus to improve Parkinsonian gait. Although skilled, automatic walking is disrupted due to basal ganglia dysfunction in Parkinson's disease, cognitive strategies seem to elicit more normal movement. ${ }^{16}$ This approach is advantageous when compared with strategies reliant on external visual cues such as lines painted on the floor or an upturned walking stick. ${ }^{34}$

The benefits of each instructional strategy may vary based on severity of Parkinson's disease and individual differences. For example, one patient with Parkinson's disease at Hoehn and Yahr stage III responded to the instruction to walk fast without changes in step length or arm swing amplitude, yet with a dramatic increase in the speed of stepping. The patient walked $114 \%$ faster, yet, the increase was achieved by a $139 \%$ increase in cadence. By contrast, the cues for deliberate arm swing and large steps resulted in more normal walking patterns with significant increases in velocity, arm swing amplitude, and step length. The large step and arm swing instructions were therefore more effective strategies. Although this patient's response to the walk fast instruction was an exception, it may be a consequence of the advanced stage of disease progression. ${ }^{6}$
In summary, following specific verbal instructions, patients with Parkinson's disease intentionally and successfully altered single gait variables including step length, velocity, and arm swing excursion. Automatically activated increases in the amplitude of other variables also occurred, producing a more normal walking pattern. Thus the use of instructional sets is a rehabilitation strategy which may assist patients with Parkinson's disease to immediately improve their movement. Notwithstanding, the benefits of instructional sets may diminish when the patient negotiates turns and corners and may therefore require self cueing to maintain gains. ${ }^{15}$ Patients with Parkinson's disease may also benefit from a practised self instructional strategy to enhance the retention of training. The effectiveness of this behavioural strategy may, however, be dependent on the progression of Parkinson's disease and the severity of locomotor disturbance.

We acknowledge and thank the people who participated in this project and who contributed to collection and analysis of the data including Osnat Teitelbaum, Patricia Moreno, and Mike Umans.

1 Murray P, Sepic SB, Gardner GM, et al. Walking patterns of men with Parkinsonism. Am $\mathcal{f}$ Phys Med 1978;60:278-94.
Knutsson E. An analysis of Parkinsonian gait. Brain 2 Knutsson E. An

3 Bagley S, Kelly B, Tunnicliffe N, et al. The effect of visual cues on the gait of independently mobile Parkinson's disease patients. Physiotherapy 1990;77:415-20.

4 Dunne JW, Hankey GJ, Edis RH. Parkinsonism: upturned walking stick as an aid to locomotion. Arch Phys Med Rehabil 1987;68:380-1.

5 Morris ME, Iansek R, Matyas TA, et al. Stride length regulation in Parkinson's disease: normalization strategies and underlying mechanisms. Brain 1996;119:551-68.

6 Teitelbaum P, Maurer RG, Fryman J, et al. Dimensions of disintegration in the stereotyped locomotion characteristic of Parkinsonism and autism. In: Sprague RL, Newell KM, eds. Stereotyped movements: brain and behavior relationships. Washington, DC: American Psychological Association, 1996:167-93.

7 Leonard C. The neurophysiology of human locomotion. In: Craik RL, Oatis CA, eds. Gait analyis: theory and application. St Louis: Mosby, 1995:46-64.

8 Craik RL, Dutterer L. Spatial and temporal characteristics of foot fall patterns. In: Craik RL, Oatis CA, eds. Gait analyis: theory and application. St Louis: Mosby, 1995:14552.

9 Folstein MF, Folstein SE, McHugh PR. Mini-mental state. Psychiatr Res 1975;12:189-98.

10 Hoehn MM, Yahr, MD. Parkinsonism: onset, progression and mortality. Neurology 1967;17:427-42.

11 Two-dimensional videography, Peak V manual, version 5.1. Englewood, Colorado: Peak Performance Technologies, 1993.

12 Gaudet G, Goodman R, Landry M, et al. Measurement of step length and step width: a comparison of videotape and direct measurements. Physiotherapy Canada 1990;42:12-5.

13 Stuber WA, Colerick VL, Blanke DJ, et al. Comparison of a clinical gait analysis method using videography and temporal distance measurements with $16 \mathrm{~mm}$ cinematography. Phys Ther 1988;68:1221-5.

14 Wall JC. Measurement of the temporal gait parameters from a videorecording. In: Woolacott M, Horak F, eds. Posture and gait: control mechanisms. Vol I. Eugene, Oregon: University of Oregon Books, 1992:440-3.

15 Morris ME, Iansek R, Summers JJ, et al. Motor control considerations for the rehabilitation of gait in Parkinson's disease. In: Glencross DJ, Piek JP, eds. Motor control and sensory motor integration: issues and directions. Amsterdam: Elsevier, 1995:61-93.

16 Iansek R, Bradshaw J, Phillips J, et al. Interaction of the basal ganglia and supplementary motor area in the elaboration of movement. In: Glencross DJ, Piek JP, eds. Motor control and sensory motor integration: issues and directions. Amsterdam: Elsevier, 1995:49-60. 